This item was submitted to Loughborough's Research Repository by the author.

Items in Figshare are protected by copyright, with all rights reserved, unless otherwise indicated.

\title{
Outerplanar crossing numbers of 3-row meshes, Halin graphs and complete p-partite graphs
}

PLEASE CITE THE PUBLISHED VERSION

PUBLISHER

(C) Springer Verlag

LICENCE

CC BY-NC-ND 4.0

REPOSITORY RECORD

Fulek, R., Hongmei He, Ondrej Sykora, and Imrich Vrt'o. 2019. "Outerplanar Crossing Numbers of 3-row Meshes, Halin Graphs and Complete P-partite Graphs”. figshare. https://hdl.handle.net/2134/2385. 
This item was submitted to Loughborough's Institutional Repository by the author and is made available under the following Creative Commons Licence conditions.

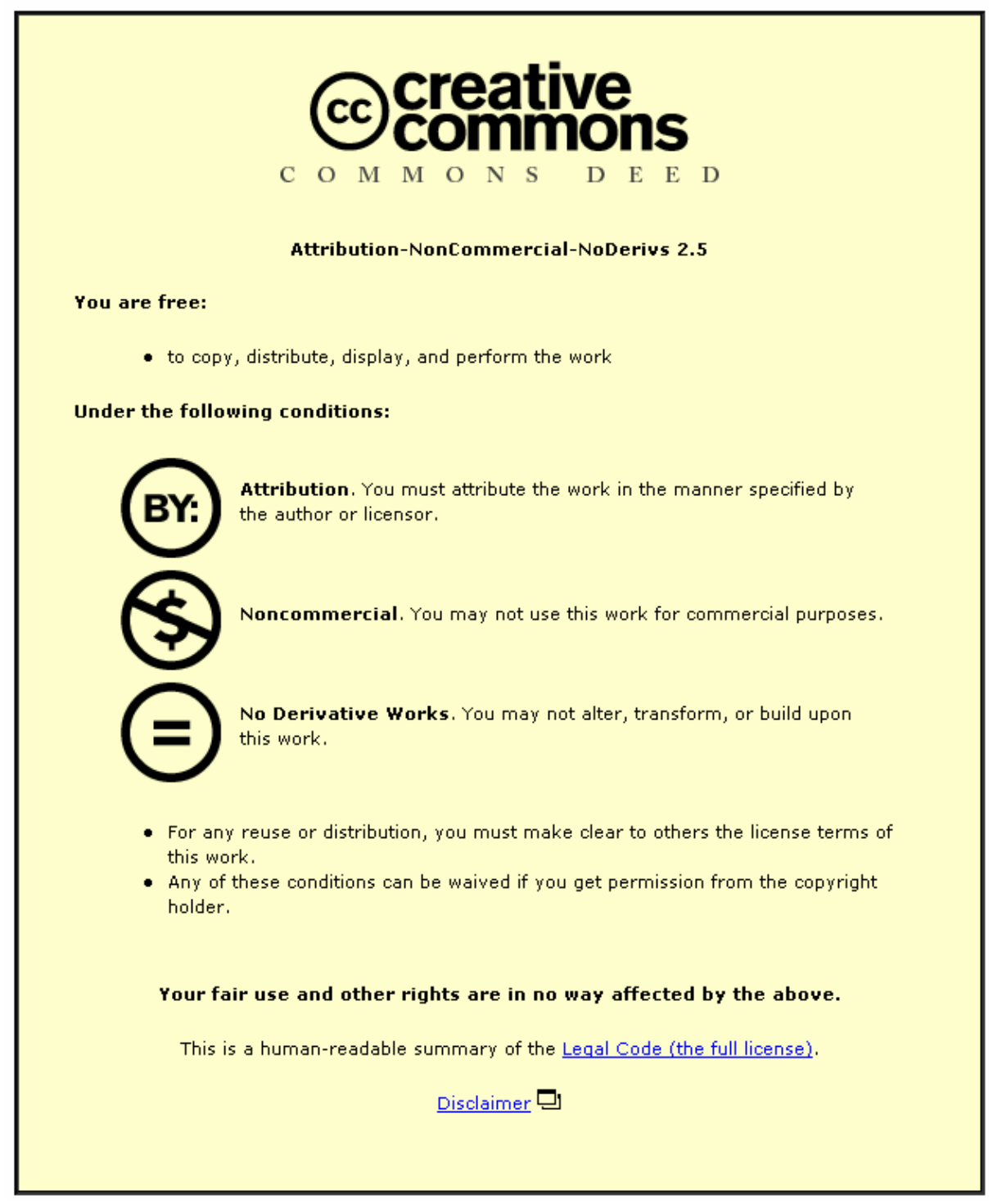

For the full text of this licence, please go to: http://creativecommons.org/licenses/by-nc-nd/2.5/ 


\title{
Outerplanar Crossing Numbers of 3-Row Meshes, Halin Graphs and Complete $p$-Partite Graphs ${ }^{\star}$
}

\author{
Radoslav Fulek ${ }^{1}$, Hongmei $\mathrm{He}^{2}$, Ondrej Sýkora ${ }^{2}$, and Imrich Vrto ${ }^{3}$ \\ 1 Department of Computer Science, Comenius University, Mlynská dolina, 84248 \\ Bratislava, Slovak Republic \\ radkofulek@pobox.sk \\ 2 Department of Computer Science, Loughborough University, Loughborough, \\ Leicestershire LE11 3TU, United Kingdom \\ $\{$ h.he, o.sykora\}@lboro.ac.uk \\ 3 Department of Informatics, Institute of Mathematics, Slovak Academy of Sciences, \\ Dúbravská 9, 84104 Bratislava, Slovak Republic \\ vrto@savba.sk
}

\begin{abstract}
An outerplanar (also called circular, convex, one-page) drawing of an $n$-vertex graph $G$ is a drawing in which the vertices are placed on a circle and each edge is drawn using one straight-line segment. We derive exact results for the minimal number of crossings in any outerplanar drawings of the following classes of graphs: 3-row meshes, Halin graphs and complete $p$-partite graphs with equal size partite sets.
\end{abstract}

\section{Introduction}

Let $G=(V, E)$ denote a graph and $\operatorname{deg}(v)$ denote the degree of $v \in V$. A drawing of a graph $G$ with vertices of the graph placed on a circle and the edges drawn as straight-line segments is called outerplanar drawing of $G$. The outerplanar crossing number $\nu_{1}(G)$ is defined as the minimum number of pairs of crossing edges over all outerplanar drawings of $G$ [1] (to find the outerplanar crossing number is NP-hard problem [2]).

We use this notation for outerplanar crossing number in accordance with the $k$-page crossing number $\nu_{k}[4,5]$ ). There are other notations and terminologies used for this quantity as circular, convex and one-page crossing number.

Let $D(G)$ denote an outerplanar drawing of $G$ and let $\nu_{1}(D(G))$ denote the number of crossings in this drawing.

The only known exact result for outerplanar crossing numbers is in 3 . It is shown for complete bipartite graphs and for example in the case that $m$

\footnotetext{
* This research was supported by the EPSRC grant GR/S76694/01 and by VEGA grant No. 2/3164/23
} 
divides $n$, it holds that $\nu_{1}\left(K_{m, n}\right)=\frac{1}{12} n(m-1)(2 m n-3 m-n)$ and in the case $m=n, \nu_{1}\left(K_{n, n}\right)=n\left(\begin{array}{l}n \\ 3\end{array}\right)$.

In this short contribution we give the propositions on outerplanar crossing numbers of the 3-row meshes, Halin graphs and complete $p$-partite, $p \geq 3$, graphs with equal sizes of the partite sets. One of the motivations for this kind of the research is to provide graphs which can be used to test heuristics for low crossing outerplanar drawings.

\section{$2 \quad$ Meshes}

Let $P_{m}$ be an $m$-vertex path. Let $P_{m} \times P_{n}$ denote the $m \times n$ mesh graph. The graph $P_{m} \times P_{n}$ is a union of $m$ paths of type $P_{n}$ (called rows) and $n$ paths of type $P_{m}$ (called columns). Let us call the subgraph consisting of edges incident to the first (last) two column vertices the comb, respectively.

Theorem 1. For any $n \geq 3$,

$$
\nu_{1}\left(P_{3} \times P_{n}\right)=\left\{\begin{array}{l}
2 n-3, \text { if } n \text { is odd } \\
2 n-4, \text { if } n \text { is even }
\end{array}\right.
$$

Proof. The order of vertices on the circle in an optimal drawing for even $n$ is given by the Hamiltonian cycle. For odd $n$ the order is same, except the last column, which follows the order of the last column in even case.

The lower bound is proved by induction on odd $n$. The cases $n=3,4,5$ are proved by using a computer. By using a computer we also proved that in any drawing of $P_{3} \times P_{4}$ every comb contains at least 4 crossings. Suppose that the claim holds for some odd $n \geq 5$. Consider an optimal outerplanar drawing of $P_{3} \times P_{n+2}$. By deleting the edges of the 4 -th,..., $(n+1)$-st column we get a graph homeomorphic to $P_{3} \times P_{4}$. we conclude that the left comb of $P_{3} \times P_{4}$ contains at least 4 crossing. It means that there are at least 4 crossings on the comb edges in the drawing of $P_{3} \times P_{n+2}$. By deleting the comb edges we get a drawing $D\left(P_{3} \times P_{n}\right)$. By the inductive assumption we have

$$
\nu_{1}\left(P_{3} \times P_{n+2}\right) \geq 4+\nu_{1}\left(D\left(P_{3} \times P_{n}\right)\right) \geq 4+2 n-3=2(n+2)-3 .
$$

Proof for even $n$ is similar.

\section{Outerplanar Crossing Numbers of Halin Graphs}

A Halin graph $G$ consists of a tree with $m$ leaves connected by a cycle, with no vertices of degree 2 except the root.

Theorem 2. For a Halin graph $G$, with a tree with $m$ leaves:

$$
\nu_{1}(G)=m-2
$$


Proof. Lower bound.

Given a drawing of $G$, number the leaves of $G$ on circle starting from root in clockwise manner as $u_{0}, \ldots u_{m-1}$. The leaves of the tree of $G$ divide the circle into $m$ intervals of internal tree vertices which are either empty or contain some vertices of the tree of $G$. Observe that any edge between vertices of two different intervals cross at least 2 edges of the cycle of $G$. Further observe that an edge incident with a leaf and a vertex from a non-neighbouring interval causes at least one crossing on an edge of the cycle. Now, consider edges incident with leaf vertices except $u_{0}$ and $u_{m-1}$. Any leaf edge except edges incident with $u_{0}$ and $u_{m-1}$ must cause at least one crossing apart from the case when it is incident with a vertex in the neighbouring intervals. Denote the vertex $v$. For every interval there are at most two such leaves. As between the vertex $v$ and the root exists a unique path in the tree, there have to be an edge in this path crossing the cycle at least twice. Thus we can assign to every leaf except $u_{0}$ and $u_{m-1}$ at least one crossing which implies at least $m-2$ crossings in any outerplanar drawing of $G$. Upper bound.

First we describe the construction of the order of the vertices. We assign a type pre, in, or post to every vertex of $G$ in the following way: the root will be of type pre, the left most child of the root will be of type pre, the right most child of the root will be of type post, rest of the children of the root will be of type $i n$. Type of $\operatorname{deg}(v)-1$ children $a_{1}, \ldots a_{d e g}(v)-1$ of a vertex $v$, except the root, is calculated as follows: if $v$ is of the type in, then the children $a_{1}, \ldots a_{\left\lfloor\frac{\operatorname{deg}(v)-1}{2}\right\rfloor-1}$ and $a_{\left\lfloor\frac{\operatorname{deg}(v)-1}{2}\right\rfloor+2}, \ldots a_{\operatorname{deg}(v)-1}$ are of type $i n, a_{\left\lfloor\frac{\operatorname{deg}(v)-1}{2}\right\rfloor}$ are of type post, $a_{\left\lfloor\frac{\operatorname{deg}(v)-1}{2}\right\rfloor+1}$ are of type pre, if $v$ is of type pre, $a_{2}, \ldots a_{\operatorname{deg}(v)-1}$ are of type $i n, a_{1}$ are of type pre, if $v$ is of type post, then the children $a_{1}, \ldots a_{\operatorname{deg}(v)-2}$ are of type $i n, a_{d e g}(v)-1$ are of type post. Define $p(v)$ the sequence of vertices of subtree with root $v$ as follows (children of $v$ are the same as above): if $v$ is of type pre then $p(v)=v, p\left(a_{1}\right), p\left(a_{2}\right), \ldots p\left(a_{\operatorname{deg}(v)-1}\right)$. If $v$ is of type post then $p(v)=p\left(a_{1}\right), p\left(a_{2}\right), \ldots p\left(a_{(\operatorname{deg}(v)-1)}\right), v$. If $v$ is of type $i n$ then $p(v)=$ $p\left(a_{1}\right), \ldots p\left(a_{\left\lfloor\frac{\operatorname{deg}(v)-1}{2}\right\rfloor}\right), v, p\left(a_{\left\lfloor\frac{\operatorname{deg}(v)-1}{2}\right\rfloor+1}\right), \ldots p\left(a_{\operatorname{deg}(v)-1}\right)$.

This drawing of the Halin graph contains exactly $m-2$ crossings. For lack of space, we skip the proof of this.

\section{Complete $p$-Partite Graphs $K_{n}(p)$}

In this section we prove an exact result for the outerplanar crossing number of complete $p$-partite graphs with equal sizes of the partite sets. Denote

$$
K_{n}(p)=K_{p}^{n, n, \ldots, n} .
$$

Theorem 3. For the complete $p$-partite graph with $n$ vertices in each partite set

$$
\nu_{1}\left(K_{n}(p)\right)=n^{4}\left(\begin{array}{l}
p \\
4
\end{array}\right)+\frac{1}{2} n^{2}(n-1)(2 n-1)\left(\begin{array}{l}
p \\
3
\end{array}\right)+n\left(\begin{array}{l}
n \\
3
\end{array}\right)\left(\begin{array}{l}
p \\
2
\end{array}\right)
$$


Proof. First we use 2 known facts shown in [3].

$$
\begin{gathered}
\nu_{1}\left(K_{n, 2 n}\right)=\frac{1}{6} n^{2}(n-1)(4 n-5) \\
\nu_{1}\left(K_{n, n}\right)=n\left(\begin{array}{l}
n \\
3
\end{array}\right)
\end{gathered}
$$

Lower bound. In every drawing of $K_{n}(p)$ there are 3 types of crossings of edges: for $i=2,3,4$, in the $i$-coloured crossing, the endvertices of the corresponding edges are coloured by $i$ colours.

The number of the 2-coloured crossings is clearly $\left(\begin{array}{l}p \\ 2\end{array}\right) \nu_{1}\left(K_{n, n}\right)$.

The number of 3 -coloured crossings is clearly at least $p\left(\begin{array}{c}p-1 \\ 2\end{array}\right)\left(\nu_{1}\left(K_{n, 2 n}\right)-\right.$ $\left.2 \nu_{1}\left(K_{n, n}\right)\right)$. We have $p$ possibilities to choose the colour $c_{1}$ which appears twice among the endvertices of a crossing and $\left(\begin{array}{c}p-1 \\ 2\end{array}\right)$ possibilities to choose two distinct colours $c_{2}$ and $c_{3}$. Then we identify the colours $c_{2}$ and $c_{3}$, which gives the total number of $\nu_{1}\left(K_{n, 2 n}\right)$ crossings. However this number contains the numbers of 2coloured crossings given by the colours $c_{1}, c_{2}$ and $c_{1}, c_{3}$ which must be subtracted.

The number of the 4-coloured crossings is $\left(\begin{array}{l}p \\ 4\end{array}\right) n^{4}$. Realize that any four vertices of distinct colours produce one 4-coloured crossing. Summing up the numbers of all 3 types of crossings and substituting (2) and (3) we get the lower bound.

Upper bound. Place the vertices of the partite sets evenly around a cycle, i.e., the vertices of every partite set form a regular $n$-gon. Then one can check, that the number of $i$-coloured crossings, for $i=2,3,4$, is the same as in the lower bound proof.

\section{References}

1. Kainen, P.C., The book thickness of a graph II, Congressus Numerantium, 71 (1990), 121-132.

2. Masuda, S., Kashiwabara, T., Nakajima, K., Fujisawa, T., On the $N P$-completeness of a computer network layout problem, in: Proc. IEEE Intl. Symposium on Circuits and Systems 1987, IEEE Computer Society Press, Los Alamitos 1987, 292-295.

3. Riskin, A., On the outerplanar crossing numbers of $K_{m, n}$, Bulletin ICA 39 (2003), 7-15.

4. Shahrokhi, F., Sýkora, O., Székely, L.A., Vrťo, I., Crossing numbers: bounds and applications, in: Intuitive Geometry, Bolyai Society Mathematical Studies 6, (I. Bárány and K. Böröczky, eds.), Akadémia Kiadó, Budapest 1997, 179-206.

5. Shahrokhi, F., Sýkora, O., Székely, L.A., Vrťo, I., The book crossing number of graphs, J. Graph Theory, 21 (1996), 413-424. 